\title{
Avaliação do nível de conhecimento de acadêmicos de Odontologia sobre emergências médicas
}

\author{
Yann Lucas Barboza*; Daniella Guedes de Figueiredo Lopes**; Celso Neiva Campos**** \\ * Residente em Cirurgia e Traumatologia Bucomaxilofacial, \\ Hospital Universitário, UEPG \\ ** Mestranda, Programa de Pós-graduação em Clínica \\ Odontológica, UFJF \\ *** Professor Titular, Departamento de Clínica Odontológica, \\ UFJF
}

Recebido: 26/09/2020. Aprovado: 11/01/2021.

\begin{abstract}
RESUMO
Emergências médicas são imprevisíveis e o cirurgião-dentista deve estar preparado para reconhecer e atuar frente a esses casos. No entanto, parece existir uma insegurança diante de tais situações, provavelmente devido às dificuldades de diagnosticar e conduzir esses eventos. Assim, esse estudo se justifica pela necessidade de avaliar o conhecimento dos acadêmicos frente a emergências médicas. O objetivo dessa pesquisa foi avaliar o nível de conhecimento sobre emergências médicas de graduandos em Odontologia. Esta pesquisa de natureza quantitativa, descritiva e exploratória, foi realizada com acadêmicos do Curso de Odontologia da Faculdade de Odontologia da Universidade Federal de Juiz de Fora, do sexto ao décimo períodos. A coleta de dados foi realizada por meio da aplicação de um teste com nove questões objetivas sobre emergências médicas. Os dados foram analisados e os resultados expressos em percentagens, médias e desvios-padrão. Os resultados mostraram que de uma amostra de 100 estudantes, sendo $21 \%$ homens e $79 \%$ mulheres, $99 \%$ têm interesse em participar de um curso de emergências médicas; $4 \%$ se julgam preparados para atuar nestes casos, $10 \%$ se consideram capazes de fazer uma reanimação cardiopulmonar e $79 \%$ obtiveram pontuação considerada como insuficiente no teste. Concluiu-se que os alunos não se mostraram preparados para atuar em casos de emergências médicas.

Descritores: Educação em Odontologia. Consultórios Odontológicos. Emergências.
\end{abstract}




\section{INTRODUÇÃO}

Emergências são situações inesperadas que exigem ações imediatas para salvar a vida e podem ocorrer a qualquer hora, em qualquer lugar e com qualquer indivíduo ${ }^{1}$. As urgências diferem das emergências quanto ao tempo para realizar tal ação, não precisando de uma resposta imediata do profissional. Este, deparando-se com uma situação de urgência, pode até se planejar e relembrar o protocolo indicado para atuar ${ }^{2}$. Em um ambiente de prática odontológica, o cirurgião-dentista $(\mathrm{CD})$ tem a responsabilidade de reconhecer e iniciar procedimentos primários de gerenciamento de emergências, em um esforço para reduzir a morbimortalidade quando esses eventos adversos surgirem ${ }^{3}$.

Entre as situações emergenciais mais comuns que ocorrem em consultórios odontológicos estão síncope, reações alérgicas, convulsões, hipoglicemia, obstrução de vias aéreas, angina pectoris, infarto do miocárdio e crise de asma $^{4-7}$. Tais quadros, geralmente, estão associados a episódios prévios de medo, ansiedade e estresse que precedem ao atendimento, e ocorrem mais comumente frente à procedimentos cirúrgicos, tratamentos endodônticos e durante ou logo após a aplicação de anestesia local ${ }^{8}$.

Os CDs devem possuir treinamento em urgências e emergências médicas (UEM), bem como domínio das diretrizes apresentadas nos protocolos estabelecidos pelo Advanced Cardiovascular Life Support (ACLS) e de reanimação cardiopulmonar $(\mathrm{RCP})^{9}$, sendo necessário fazer uma reciclagem a cada dois anos ${ }^{10}$. Contudo, nos cursos de graduação e de pósgraduação, em geral, observa-se que, quando oferecido, o treinamento é direcionado para manobras de suporte básico (que de acordo com os atuais protocolos são direcionadas a leigos, apresentando uma abordagem diferente de capacitação do indivíduo) e, além disso, a capacitação é realizada de forma curta ou deficiente. Dessa forma, o CD deve buscar por si só tal conhecimento, uma vez que a Lei 5081/6623, que regulamenta o exercício da Odontologia, afirma que o profissional deve prescrever e aplicar medicação de urgência no caso de acidentes graves que comprometam a vida, e o Código Penal determina que omitir socorro à vítima de acidentes ou pessoas em perigo iminente, podendo fazê-lo, pode ser considerado crime ${ }^{1}$.

As UEM estão cada vez mais frequentes no cotidiano dos CDs, devido, sobretudo, ao aumento gradativo da proporção de pacientes comprometidos sistemicamente que utilizam os serviços odontológicos, o que torna perceptível a falta de formação e negligência quanto ao arsenal necessário para reverter situações de emergência ${ }^{11}$. Muitos CDs se preocupam mais com a melhoria de sua técnica e esquecem de olhar o paciente como um todo, revelando uma formação tecnicista da classe $^{1}$.

Acadêmicos e profissionais se mostram inseguros para diagnosticar e tratar emergências médicas ${ }^{2,4,12,13}$. O despreparo para atuar em casos de parada cardiorrespiratória também é evidente, sendo necessária a revisão continua das técnicas para evitar o seu esquecimento ${ }^{14,15}$.

Assim, este estudo objetivou avaliar o nível de conhecimento sobre emergências médicas de graduandos em Odontologia.

\section{MATERIAL E MÉTODO}

Trata-se de estudo de natureza quantitativa, descritiva e exploratória, aprovado pelo Comitê de Ética em Pesquisa da Universidade Federal de Juiz de Fora (UFJF), em Minas Gerais, sob o parecer número 2.491.649. O instrumento de pesquisa foi elaborado pelos pesquisadores com base em questionários aplicados em estudos anteriores $^{1,2,4,6,7,10,11,17}$, respondido de forma presencial, sem prévio aviso. Como respondentes, participaram alunos do sexto ao décimo períodos, sendo que todos já haviam 
trabalhado o conteúdo de emergências médicas, tema que é abordado no quinto período do curso, na disciplina de anestesiologia.

O questionário abordou oito questões direcionadas a aspectos sociodemográficos, sendo elas de múltipla escolha, do tipo "sim ou não", acerca da preocupação com capacitação em UEM e autopercepção de habilidades para diagnóstico e tratamento das mesmas (figura 1). Em relação ao conhecimento sobre emergências médicas foram aplicadas nove questões de múltipla escolha, baseadas em diagnóstico (3 questões) e tratamento de UEM (6 questões) (figura 2). Juntamente com o questionário, foi entregue aos participantes um Termo de Consentimento Livre e Esclarecido, explicando sobre a pesquisa e assegurando a privacidade e o sigilo dos dados disponibilizados.

Os dados coletados foram tabulados em planilha eletrônica Excel ${ }^{\circledR}$ (Microsoft Corp., Redmond, WA, EUA) e analisados estatisticamente por meio do software SPSS $^{\circledR}$ (IBM, Armonk, NY, EUA), utilizando-se médias e desvios-padrão, para categorizar os dados descritivos. O teste t de Student foi empregado para comparar o domínio do assunto pelos alunos do sexto período, imediatamente após a conclusão da disciplina, aos demais.

1. Período: 2 Sexo: ( ) Masculino ( ) Feminino $\quad$ 3. Idade: _ anos
4. Cor que se declara: ( ) Branco ( ) Pardo ( ) Amarelo ( ) Negro ( ) Indígena
5. Participa ou já participou de algum curso extracurricular sobre emergência médicas? ( ) Sim ( ) Não
6. Você gostaria de participar de um curso sobre emergência médicas em Odontologia? ( ) Sim ( ) Não
7. Você se sente preparado para atuar em um caso de emergência médica? ( ) Sim ( ) Não
8. Você se julga capaz de realizar uma reanimação cardiopulmonar? ( ) Sim ( ) Não

Figura 1. Questionário sociodemográfico

\section{RESULTADOS}

Dos 192 estudantes matriculados nos períodos especificados, $100(52,1 \%)$ concordaram em participar da pesquisa (sete não responderam ao questionário integralmente), sendo 21 (21\%) deles do sexo masculino e 79 (79\%) do sexo feminino. A idade dos participantes variou entre 19 e 28 anos, com média de 22,47anos.

Declararam já ter participado de algum curso extracurricular sobre UEM $28 \%$ dos alunos. Ainda assim, 99\% dos participantes do estudo manifestaram o desejo em participar de um curso sobre emergências médicas; apenas $4 \%$ dos respondentes se sentem preparados para atuar em um caso de UEM e 10\% se consideram capazes de realizar manobras de reanimação cardiopulmonar.

Dos participantes, $7 \%$ não declararam o período do curso; havendo maior representação do nono, com 25 alunos. O oitavo período foi o que teve menor número de participantes, com 15 alunos. O sexto, sétimo e décimo períodos tiveram, respectivamente, 18, 17 e 18 respondentes.

Alunos que obtiveram 9 acertos foram considerados com performances muito boas. $\mathrm{O}$ desempenho foi considerado bom por aqueles que acertaram 8 questões e, aqueles que atingiram um número de acertos inferior a esse valor foram classificados como regular e abaixo das expectativas, respectivamente. Apenas dois participantes alcançaram o valor máximo de nove acertos; $9 \%$ obtiveram 8; $10 \%$ apresentaram sete acertos e os 79 respondentes restantes ficaram abaixo do considerado ideal. Os resultados encontram-se na tabela 1. A tabela 2 e indica quantos participantes acertaram cada questão e em qual período do curso se encontra. 
1. Um paciente apresentou durante a consulta os seguintes sinais e sintomas: perda temporária e momentânea da consciência, hipotensão, palidez, taquicardia, escurecimento da visão e sonolência. Qual o diagnóstico?

a) Lipotimia; b) Síncope vasovagal; c) Crise hipertensiva; d) Hipoglicemia.

2. Qual a conduta para tratar o caso apresentado na questão acima?

a) Dar para o paciente ingerir carboidratos simples (refrigerantes, doces, ...) ou glicose gel (15-24 mg). Se não houver melhora ou o paciente ficar inconsciente, administrar $50 \mathrm{ml}$ de solução aquosa de glicose a $50 \%$ (dextrose) por via endovenosa durante dois a três minutos;

b) Iniciar técnicas de ressuscitação cardiopulmonar;

c) Interromper o atendimento, colocar o paciente em posição confortável, monitorar sinais vitais, tranquilizálo e administrar captopril ( $25 \mathrm{a} 50 \mathrm{mg}$ ) por via sublingual;

d) Colocá-lo em posição supina com os membros inferiores levemente elevados em relação à cabeça (10 a 15 graus) e estender a cabeça para trás, aguardando 2 a 3 minutos. Caso não ocorra melhora deve-se administrar oxigênio ( 2 a 4 litros/minuto) e monitorar sinais vitais enquanto aguarda auxílio médico previamente solicitado

3. Após anestesia, durante o procedimento cirúrgico, seu paciente apresentou dor de cabeça, tontura, mal estar, confusão mental, distúrbio visual e hemorragia gengival após a manipulação. Qual o diagnóstico?

a) Crise hipertensiva; b) Acidente vascular encefálico; c) Angina pectoris; d) Infarto do miocárdio

4. Qual a medicação de escolha para tratar um choque anafilático?

a) Captopril; b) Midazolan; c) Dexametasona; d) Adrenalina.

5. Qual conduta clínica frente a um episódio de crise convulsiva?

a) Colocar o paciente em posição supina com membros inferiores levemente elevados em relação à cabeça (10 a 15 graus) e estender a cabeça para trás, aguardando 2 a 3 minutos. Caso não ocorra melhora deve-se administrar oxigênio ( 3 a 4 litros/minuto) e monitorar sinais vitais enquanto se aguarda auxílio médico previamente solicitado;

b) Interromper o atendimento, colocar o paciente em posição confortável e administrar oxigênio. Em seguida, administrar $5 \mathrm{mg}$ de dinitrato de isossorbida via sublingual. Caso sintomas não cedam, administra-se uma segunda dose;

c) Interromper o atendimento, remover os objetos da boca do paciente, colocá-lo em posição supina e inclinar sua cabeça para o lado. Monitorar os sinais vitais e aguardar o fim da crise. Se necessário administrar midazolam e diazepam $(0,2$ e $0,3 \mathrm{mg}$ por quilograma via intramuscular ou 5 a $10 \mathrm{mg}$ via intravenosa);

d) Iniciar técnicas de ressuscitação cardiopulmonar.

6. Como você ajudaria um paciente, em seu consultório, com hipoglicemia?

a) Interromper o atendimento, dar ao paciente carboidratos simples, caso ele esteja consciente. Mas se ele estiver inconsciente, administra-se 50 mililitros de solução aquosa de glicose a $50 \%$ por via endovenosa de 2 a 3 minutos;

b) Acabar com o procedimento o mais rápido possível; dar ao paciente polissacarídeos, caso ele esteja consciente. Mas, se ele estiver inconsciente, administra-se 50 mililitros de solução aquosa de glicose a 70\% por via endovenosa durante 5 a 7 minutos;

c) Interromper o atendimento; dar ao paciente polissacarídeos, caso ele esteja consciente. Mas se ele estiver inconsciente, administra-se 50 mililitros de solução aquosa de glicose a $70 \%$ por via endovenosa durante 5 a 7 minutos;

d) Interromper o atendimento; dar ao paciente carboidratos simples, caso ele esteja consciente. Mas, se ele estiver inconsciente, administra-se 100 mililitros de solução aquosa de glicose a 50\% por via endovenosa durante 2 a 3 minutos;

7. Qual a emergência médica mais comum em um consultório odontológico?

a) Angina pectoris; b) Lipotímia/síncope; c) Crise hipertensiva; d) Hipoglicemia

8. Qual o recurso mais importante em um kit de emergência médica?

a) Oxigênio; b) Diazepan; c) Adrenalina; d) Dexametasona.

9. Qual o segundo recurso mais importante em um kit de emergência médica?

a) Oxigênio; b) Diazepan; c) Adrenalina; d) Dexametasona.

Figura 2. Questionário para avaliar o conhecimento dos acadêmicos sobre emergências médicas em consultório odontológico 
Tabela 1. Distribuição do número de acertos para cada questão, por período

\begin{tabular}{cccccccc}
\hline $\begin{array}{c}\text { Número de } \\
\text { acertos }\end{array}$ & $\mathbf{6}^{\circ}$ & $\mathbf{7}^{\mathbf{0}}$ & $\mathbf{8}^{\mathbf{0}}$ & $\mathbf{9}^{\mathbf{0}}$ & $\mathbf{1 0}^{\mathbf{0}}$ & Não informado & Porcentagem de alunos \\
\hline $\mathbf{1}$ & - & - & - & 1 & - & - & $1 \%$ \\
$\mathbf{2}$ & 1 & - & - & - & - & 1 & $2 \%$ \\
$\mathbf{3}$ & - & 1 & 2 & 2 & 1 & - & $6 \%$ \\
$\mathbf{4}$ & - & 3 & 3 & 5 & 4 & 1 & $16 \%$ \\
$\mathbf{5}$ & 5 & 4 & 2 & 6 & 5 & 3 & $25 \%$ \\
$\mathbf{6}$ & 7 & 5 & 4 & 6 & 5 & 2 & $10 \%$ \\
$\mathbf{7}$ & 3 & 2 & 2 & 2 & 1 & - & $9 \%$ \\
$\mathbf{8}$ & 2 & 1 & 1 & 3 & 1 & - & $2 \%$ \\
$\mathbf{9}$ & - & 1 & 1 & & 1 & - & $100 \%$ \\
\hline Total & 18 & 17 & 15 & 25 & 18 & 7 & \\
\hline
\end{tabular}

Tabela 2. Total de alunos que responderam corretamente cada questão

\begin{tabular}{cccccccc}
\hline Questão & $\mathbf{6}^{\mathbf{0}}$ & $\mathbf{7}^{\mathbf{o}}$ & $\mathbf{8}^{\mathbf{o}}$ & $\mathbf{9}^{\mathbf{0}}$ & $\mathbf{1 0}^{\mathbf{o}}$ & Não informado & $\begin{array}{c}\text { Porcentagem de } \\
\text { acertos/questão }\end{array}$ \\
\hline $\mathbf{1}$ & 5 & 9 & 4 & 10 & 3 & 1 & 32 \\
$\mathbf{2}$ & 12 & 11 & 12 & 16 & 15 & 5 & 71 \\
$\mathbf{3}$ & 12 & 13 & 11 & 17 & 16 & 5 & 73 \\
$\mathbf{4}$ & 16 & 13 & 11 & 18 & 10 & 5 & 97 \\
$\mathbf{5}$ & 18 & 16 & 15 & 23 & 18 & 7 & 78 \\
$\mathbf{6}$ & 14 & 15 & 8 & 19 & 16 & 6 & 57 \\
$\mathbf{7}$ & 6 & 9 & 12 & 16 & 11 & 3 & 34 \\
$\mathbf{8}$ & 14 & 5 & 4 & 5 & 5 & 1 & 30 \\
$\mathbf{9}$ & 9 & 5 & 5 & 7 & 4 & - & \\
\hline
\end{tabular}

A questão com maior índice de acerto foi a de número 5 com $97 \%$, que se referia a conduta clínica a ser realizada diante de uma crise convulsiva. A nona questão, que se referia ao conhecimento relacionado ao conteúdo do kit de emergência médica, foi a que apresentou menos acertos, onde apenas 30 pessoas acertaram a resposta.

O sexto período foi usado como base para comparação com os demais, visto que era o período mais próximo do conteúdo ministrado, esperando-se maior domínio sobre o tema, o que não se mostrou verdadeiro, pois o teste $\mathrm{t}$ de Student não evidenciou diferença significativa de desempenho entre os grupos ( $p>0,05)$.

\section{DISCUSSÃO}

As UEM não são ocorrências frequentes em consultórios odontológicos. No entanto, fatores como o aumento de pessoas idosas que procuram atendimento odontológico, terapias utilizadas, consultas odontológicas mais longas e os novos medicamentos utilizados na Odontologia podem levar a um aumento na probabilidade da ocorrência de tais complicações ${ }^{16}$, fazendo necessário um conhecimento maior dos profissionais de Odontologia sobre o tema.

Nesse sentido, o estudo em questão justifica- 
se por avaliar como o assunto está inserido na instituição onde foi aplicado e comparar seus resultados a outras instituições de ensino, com finalidade de que, a partir dos resultados obtidos, possa se elaborar estratégias para aplicação de uma melhor metodologia e exposição do assunto, como a obrigatoriedade de uma disciplina específica ou cursos periódicos de curta duração, proporcionando uma formação mais completa aos alunos, além de garantir maior aptidão e segurança aos futuros profissionais diante de quadros de UEM.

Os resultados obtidos no estudo em questão sugerem restrito conhecimento dos alunos sobre o tema, uma vez que a análise dos resultados apresentados mostra que o percentual médio de acerto entre todos os participantes foi de $61,54 \%$, revelando pouco conhecimento sobre o assunto. Esse resultado foi próximo ao encontrado por Queiroga et $a l .{ }^{4}$, que observaram acerto de $60,27 \%$ na amostra pesquisada. No presente estudo os acadêmicos do nono período foram os que apresentaram menor índice de aproveitamento (58,3\%), enquanto os do décimo período mostraram melhor desempenho (60,4\%). Essas taxas de aproveitamento estão próximas daquelas apresentadas por Queiroga et l. $^{4}$, porém com inversão entre o nono e décimo períodos $(54,5 \%$ e $66 \%$, respectivamente).

Comparando os percentuais de acerto deste estudo com os de Queiroga et al. ${ }^{4}$, tem-se, respectivamente, $65,4 \%$ e $55,6 \%$ para os sextos períodos, $62,8 \%$ e $58 \%$ para os sétimos e $60,8 \%$ e 65,3 para os oitavos. Já, um estudo realizado por Somaraj et $a .^{3}$ constatou que $39,89 \%$ dos estagiários em Odontologia possuíam conhecimento sobre UEM abaixo da média. Tais resultados demonstram a necessidade de uma abordagem mais efetiva do tema nos cursos de Odontologia.

A insegurança dos alunos em relação à atuação em caso de UEM foi observada por
Queiroga et $a .^{4}$, pois $70,3 \%$ dos alunos não se sentiam preparados para atuar em uma UEM. No presente estudo, $96 \%$ dos alunos não se consideravam aptos a atuar em situações emergenciais. No estudo de Albelaihi et al. ${ }^{12}$ apenas $37 \%$ dos participantes estavam confiantes para lidar com qualquer emergência médica no consultório odontológico.

Os alunos participantes deste estudo não receberam treinamento de RCP na graduação. Quando questionados sobre como percebiam sua capacidade de realizar uma RCP, 89,8\% deles disseram não se sentir capazes e $2 \%$ não responderam. Chapman ${ }^{17}$ observou que $64 \%$ dos acadêmicos haviam recebido treinamento em RCP na graduação, no entanto $77 \%$ não se sentiam preparados para realizá-la. Al-Iryani et al. ${ }^{15}$ relataram que $62 \%$ dos participantes não estavam confiantes para realizar RCP. Em contrapartida, Albelaihi et al. ${ }^{12}$ constataram que $71 \%$ dos estudantes e estagiários sabiam sobre a correta localização anatômica para compressão torácica. $\mathrm{O}$ conflito quanto à disponibilização do ensino, seguida pela proximidade dos dados relativos à confiança em atuar pode, talvez, ser justificado pela falta de treinamento prático contínuo, sugerindo a carência de um treinamento eficiente para a tal situação, o que torna imprescindível sua inserção curricular.

$\mathrm{Na}$ primeira questão observou-se que $32 \%$ dos alunos reconheciam os sinais e sintomas descritos para síncope vasovagal e que $49 \%$ deles responderam que se tratava de uma lipotímia. Hanna et al. ${ }^{5}$ encontraram resultado semelhante, com $35,7 \%$ dos entrevistados apontando corretamente que se tratava de um quadro de síncope vasovagal. Este resultado pode sugerir um equívoco apresentado pelos acadêmicos devido à semelhança e proximidade dos conceitos, sendo talvez necessária a adoção de estratégias que permitam diferenciá-los claramente.

Apesar da dificuldade demonstrada pelos 
acadêmicos para diferenciar síncope e lipotimia, $71 \%$ souberam o modo correto de proceder frente à situação, o que pode ser atribuído ao fato do protocolo de tratamento adotado para ambos ser o mesmo $^{18}$. Ao contrário, Silva ${ }^{19}$ apontou que apenas $28,1 \%$ da amostra sabia tratar o caso. Albelaihi et $a l .{ }^{12}$ constataram a síncope como a emergência médica mais relatada (28\%). Contudo, menos da metade dos participantes tinha conhecimento sobre o correto manejo do paciente.

Em uma descrição de caso de crise hipertensiva, $74 \%$ dos acadêmicos souberam reconhecer o episódio, diferindo do afirmado por Hanna et al. ${ }^{5}$ que encontraram um índice de acerto de $21,9 \%$ para o mesmo caso, porém com nove alternativas. A redução para quatro alternativas aumente a chance de acerto por intuição.

Quanto à medicação de escolha para tratar o choque anafilático, $73 \%$ responderam acertadamente que se tratava de adrenalina, enquanto que os resultados de Hanna et al. ${ }^{5}$ mostraram 52,4\%. Novamente, a variação no questionário pode ter sido responsável pelas diferenças de resultados. Nesta pesquisa, apenas foi questionado qual medicamento usar, entretanto, Hanna et al..$^{5}$, em duas alternativas, diferenciaram a quantidade de adrenalina a ser aplicada. A associação das duas alternativas que abordavam a adrenalina como primeira escolha levaria a um índice de $80 \%$, mais próximo do resultado desse estudo. Albelaihi et al. ${ }^{12}$ constataram que apenas $33 \%$ utilizariam adrenalina.

A avaliação da conduta frente a uma crise convulsiva, questão com maior índice de acertos neste estudo, revelou que $97 \%$ dos alunos sabem conduzir o caso, o que se mostrou presente também no estudo de Hanna et al..$^{5}$, com 80,2\%. Tais achados mostram-se como um fator positivo, uma vez que a ocorrência de uma crise convulsiva durante o tratamento odontológico deve ser controlada pelo $\mathrm{CD}$, estando esse apto a realizar etapas primárias, como a imediata interrupção do procedimento e posicionamento do paciente em decúbito lateral para diminuir a chance de aspiração de secreções ou materiais dentários ${ }^{20}$.

Um caso de hipoglicemia também foi proposto no questionário e $78 \%$ souberam responder a conduta a seguir para reverter o quadro. Em sua pesquisa, Silva ${ }^{19}$ observou que $59,5 \%$ sabiam gerenciar um caso como o citado.

Uma questão se referia sobre qual UEM era mais prevalente em consultório odontológico. Dos participantes, $57 \%$ acertaram que se tratava de síncope/lipotimia. Soramaj et al. ${ }^{3}$ obtiveram como resposta a síncope $(93,98 \%)$, convulsões $(2,73 \%)$, reação anafilática e reações alérgicas $(0,53 \%)$. Oliveira $^{21}$ conduziu uma pesquisa em Portugal e observou um índice de acerto de $37 \%$, já que boa parte dos entrevistados $(31,1 \%)$ optaram por hiperventilação e outra parte $(28,6 \%)$ por reações alérgicas. Assim, a forma de expor a questão pode ter interferido no processo de escolha, já que as duas outras também são situações comuns nas UEM em consultório odontológico e não figuravam no questionário deste artigo.

A disponibilidade de medicamentos de emergência no consultório odontológico é de extrema importância, sendo necessário o preparo de um kit composto por medicamentos considerados essenciais ${ }^{8}$. As duas últimas questões do presente estudo foram direcionadas para avaliação do conhecimento referente aos recursos farmacológicos mais importantes deste kit no consultório odontológico. Segundo Oliveira ${ }^{21}$ é a adrenalina, que foi reconhecida por $59,3 \%$ dos alunos. No presente estudo apenas $34 \%$ dos respondentes acertaram a questão. A última questão, em que se obteve menor índice de acertos evidenciou que $30 \%$ dos participantes apontaram o oxigênio como sendo o segundo recurso mais importante presente no kit. Tendo em vista que são dados como recursos essenciais o oxigênio, a adrenalina, a nitroglicerina, anti-histamínicos injetáveis, salbutamol, aspirina, carboidratos orais 
e os equipamentos importantes que devem estar presentes no consultório como, esfigmomanômetro, estetoscópio, seringas e agulhas e, dentro do possível, um desfibrilador externo automático ${ }^{8}$, os resultados encontrados demostram um escasso conhecimento dos alunos sobre a montagem de kits de emergência.

Outro aspecto não abordado no presente estudo foi relacionado ao atendimento infantil. Levando-se em conta que a maior parte da literatura produzida é relacionada a pacientes adultos, os CD que atendem crianças e adultos devem se preocupar em manter no consultório recursos para os dois públicos. Diferenças estruturais, assim como psicológicas, respiratórias, cardiovasculares e do sistema imunológico devem ser levadas em consideração para evitar o despreparo material e teórico para ajudar o paciente $^{22}$.

A necessidade de preparo específico para atuar em UEM é visível, assim como o desejo dos acadêmicos, visto que $99 \%$ da amostra se disse interessada em fazer um curso sobre o tema. Índice próximo ao encontrado por $\operatorname{Silva}^{19}(99,2 \%)$ e Somaraj et al. $^{3}$ (98,36\%). Nesse sentido, os resultados obtidos no presente estudo corroboram com os de outras pesquisas, evidenciando a necessidade de que os temas de suporte básico à vida e emergências em consultórios devem ser obrigatórios e transversais no currículo do ensino odontológico $^{12,15}$.

\section{CONCLUSÃO}

Mesmo com o conteúdo de UEM ministrado, os estudantes não apresentaram conhecimento suficiente para atuar diante de situações de emergências médicas, fato que pode acarretar sério risco para aqueles que procurarem seus serviços e, porventura, passem por tal situação. Assim, fica a reflexão sobre a forma mais adequada para abordar o assunto: uma disciplina específica e/ou cursos periódicos de curta duração.

\section{ABSTRACT \\ Knowledge level assessment of Dentistry undergraduate students about medical emergencies}

Medical emergencies are unpredictable and the dental surgeon must be prepared to recognize and act upon these cases. However, there seems to be insecurity before such situations, probably due to difficulties in diagnosing and conducting these events. Thus, this study is justified by the need to assess the knowledge of dental students before medical emergencies. The aim of this research was to evaluate the knowledge level of undergraduate dentistry students about medical emergencies. This quantitative, descriptive and exploratory research was performed with students from the Dentistry Course at the Dentistry School of the Federal University of Juiz de Fora, from the sixth to the tenth course terms. Data collection was accomplished through the application of a test with nine objective questions on medical emergencies. The data were analysed and the results were expressed as percentages, means and standard deviations. The results showed that out of a sample of 100 students, $21 \%$ men and $79 \%$ women, $99 \%$ are interested in participating in a medical emergency course; 4\% consider themselves prepared to act in these cases, $10 \%$ consider themselves capable of performing cardiopulmonary resuscitation and $79 \%$ obtained a score considered as insufficient in the test. It was concluded that the students did not seem prepared to act in cases of medical emergencies. Descriptors: Education, Dental. Dental Offices. Emergencies.

\section{REFERÊNCIAS}

1. Caputo IGC, Bazzo GJ, Silva RHA da, Júnior ED. Vidas em risco: emergências médicas em consultório odontológico. Rev Cir Traumatol Buco-Maxilo-Fac. 2010;10(3):51-8.

2. Haese RDP, Cançado RP. Urgências e emergências médicas em odontologia: avaliação da capacitação e estrutura dos 
consultórios de cirurgiões-dentistas. Rev Cir Traumatol Buco-Maxilo-Fac. 2016;16(3):31-9.

3. Somaraj V, Shenoy RP, Panchmal GS, Jodalli PS, Sonde L, Karkal R. Knowledge, attitude and anxiety pertaining to basic life support and medical emergencies among dental interns in Mangalore City, India. World J Emerg Med. 2017;8(2):131-5.

4. Queiroga TB, Gomes RC, Novaes MM, Marques JLS, Santos KSA, Grempel RG. Situações de emergências médicas em consultório odontológico. Avaliação das tomada de decisões. Rev Cir Traumatol BucoMaxilo-Fac. 2012;12(1):115-22.

5. Hannal LMO, Alcântra HSC, Damasceno JM, Santos MTBR. Conhecimento dos Cirurgiões Dentistas diante Urgência/Emergência Médica. Rev Cir e Traumatol Buco-maxilofac. 2014;14(2):79-80.

6. Resende RG, Lehman LFC, Miranda BB, Cunha JF, Costa MLT, Gomez RS, et al. Complicações sistêmicas no consultório odontológico: parte I. Arq Odontol. 2009; 45(1):44-50.

7. Resende RG de, Lehman LFC, Viana ACD, Alves FF, Jorge $\mathrm{KO}$, Fraga $\mathrm{MG}$, et al. Complicações sistêmicas no consultório odontológico: parte II. Arq Odontol. 2009; 45(2):93-8.

8. Haas DA. Management of medical emergencies in the dental office: conditions in each country, the extent of treatment by the dentist. Anesth Prog. 2006;53(1):20-4.

9. American Heart Association. Destaques das diretrizes de RCP e ACE de 2020 da American Heart Association. 2020, 31p.

10. Fabris V, Junqueira JLC, Silva, Mallmann F, Oliveira GR, Lucas A. Avaliação do Conhecimento dos Cirurgiões-Dentistas sobre Suporte Básico de Vida Frente a Emergências Médicas em Odontologia. J Oral Invest. 2015; 4(2):50-6.

11. Fiuza MK, Balsan ST, Pretto JLB, Cenci RA, Conto F. Avaliação da prevalência e do grau de conhecimento do cirurgião-dentista em relação às emergências médicas. RFO UPF. 2013;18(3):295-301.

12. Albelaihi HF, Alweneen AI, Ettish A,
Alshahrani FA. Knowledge, attitude, and perceived confidence in the management of medical emergencies in the dental office: A survey among the dental students and interns. J Int Soc Prev Community Dent. 2017;7(6):364-9.

13. Moretto MJ, Menezes LB, Barbieri G, Menezes PR. Emergências médicas em consultório odontológico. J Multidiscipl Dent. 2020;10(1):9-13.

14. Colet D, Griza GL, Fleig C do N, Conci RA, Sinegalia AC. Acadêmicos e profissionais da odontologia estão preparados para salvar vidas? RFO UPF. 2011;16(1):25-9.

15. Al-Iryani GM, Ali FM, Alnami NH, Almashhur SK, Adawi MA, Tairy AA. Knowledge and Preparedness of dental practitioners on management of medical emergencies in Jazan Province. Open Access Maced J Med Sci. 2018;6(2):402-5.

16. Stafuzza TC, Carrara CFC, Oliveira FV, Santos CF, Oliveira TM. Evaluation of the dentists' knowledge on medical urgency and emergency. Braz Oral Res. 2014; 28(1):1-5.

17. Chapman PJ. Medical emergencies in dental practice and choice of emergency drugs and equipment: a survey of Australian dentists. Aust Dent J. 1997;42(2):103-8.

18. Lúcio PSC, Barreto RDC. Emergências médicas no consultório odontológico e a (in)segurança dos profissionais. RBCS. 2012;16(2):267-72.

19. Silva EL. Avaliação do nível de conhecimento do uso de protocolos de urgência e/ou emergência médica na clínica odontológica. [Dissertação de Mestrado]. Belém: Centro de Ciências da Saúde - Universidade Federal do Pará: 2006. 71p.

20. Mehmet Y, Senem Ö, Sülüm T, Hümeyra K. Management of epileptic patients in Dentistry. Surg Sci. 2012; 3:47-52.

21. Oliveira VCM. Emergências médicas no consultório dentário - análise dos conhecimentos e atuação do médico dentista e futuro médico dentista. [Dissertação de Mestrado]. Almada - Portugal: Instituto Superior de Ciências da saúde Egas Moniz: 2014. 111p. 
22. Vranić DN, Jurković J, Jeličić J, Balenović A, Stipančić G, Čuković-Bagić I. Hitna stanja u dječjoj stomatologiji. Acta Stomatol Croat. 2016;50(1):72-80.
Correspondência para:

Daniella Guedes de Figueiredo Lopes

e-mail: daniellagflopes@gmail.com

Universidade Federal de Juiz de Fora

Rua José Lourenço Kelmer, S/N

36036-900 Juiz de Fora/MG 